\title{
One-dimensional metal hydroxide nanomaterials with macroscopically controlled orientation and aggregation: fascinating surface hydroxyl groups on anisotropic structures for functionalities
}

\author{
Kenji OKADA ${ }^{1,2, \dagger}$ \\ ${ }^{1}$ Department of Materials Science, Graduate School of Engineering, Osaka Prefecture University, Sakai 599-8531, Japan \\ ${ }^{2}$ JST, PRESTO, 4-1-8 Honcho, Kawaguchi, Saitama 332-0012, Japan
}

This review briefly describes recent advances on the fabrication methods affording macroscopically controlled orientation and aggregation of one-dimensional (1D) metal hydroxide nanomaterials and the structuralizationdriven functional applications. A special attention is focused on the functionalization based on two approaches: 1) superhydrophobic adhesive surface, and monolithic cation-exchangers and photocatalysts by controlled orientation and aggregation of titanate nanotubes, 2) a supported catalyst, electrothermal sensor and switchable fluorescence film by the structuralization of metal organic framework (MOF) derived from orientation/ aggregation-controlled copper hydroxide nanotubes/nanobelts.

(C)2020 The Ceramic Society of Japan. All rights reserved.

Key-words: Metal hydroxide, ID nanomaterial, Titanate nanotube, Porous material, MOF, Orientation, Aggregation, Film, Hierarchical structure, Epitaxial

[Received April 28, 2020; Accepted June 9, 2020]

\section{Introduction}

Nanomaterials have been used in a wide range of fields including electrical, optical, environmental and pharmaceutical applications due to the unique properties such as a large specific surface area and quantum effect in contrast to bulk materials. ${ }^{1)}$ Among various morphologies of nanomaterials, one-dimensional (1D) nanomaterials have attracted attentions for potential applications including electronics, optoelectronics and advanced coatings due to their anisotropic physical and chemical properties derived from their structural anisotropy. The performance of the materials is closely related to not only their size and shape but also macroscopic aggregation states (positioning the 1D nanomaterials at the desired places) and orientation of individuals. $^{2)}$

Metal hydroxides, constructed by a reaction between hydroxide anions and metal cations in basic aqueous media, are mostly obtained as crystalline nanomaterials with 1D or 2D structures, of which crystal structures are composed of a stacking of single metal hydroxide layers by hydrogen bonding or intercalating cation/anion species (Fig. 1). Metal hydroxides exhibit unique features: 1) aligned and abundant hydroxyl groups on their surfaces,

Corresponding author: K. Okada; E-mail: k_okada@mtr. osakafu-u.ac.jp
2) low lattice enthalpy compared to metal oxides, and 3) a layered structure capable of ion-exchange, which are different from metal oxides. Although these unique features allow metal hydroxide nanomaterials to be utilized in a variety of applications, ${ }^{3)}$ especially as catalysts, ${ }^{4)}$ adsorbents, ${ }^{5), 6)}$ and ion-exchangeable materials, ${ }^{7}$ ) fascinating applications derived from the above-mentioned nature of metal hydroxides have not been fully explored yet. For instance, to our best knowledge, an efficient use of their aligned hydroxyl groups on their surfaces have not been studied. A control of macroscopic orientation and aggregation of metal hydroxide nanomaterials will open up the possible utilization of metal hydroxide nanomaterials for specific applications, as demonstrated in other 1D nanomaterials such as carbon nanotube and others. In this review, fabrication methods affording macroscopic orientation and aggregation of 1D metal hydroxide nanomaterials and their functional applications achieved by utilizing the features of 1D metal hydroxides are overviewed with an emphasis on the author's works (Fig. 2). The functionalization was achieved by two approaches: 1) controlled orientation and aggregation of 1D titanate nanotubes, 2) the structuralization of other functional materials, metal organic framework (MOF), derived from orientation/ aggregation-controlled copper hydroxide nanotubes/nanobelts. The two different approaches are briefly reviewed in the following sections. 
(a) Nanostructures
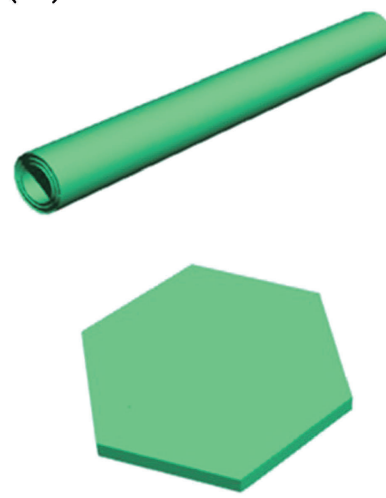

(b)

\section{Surface}

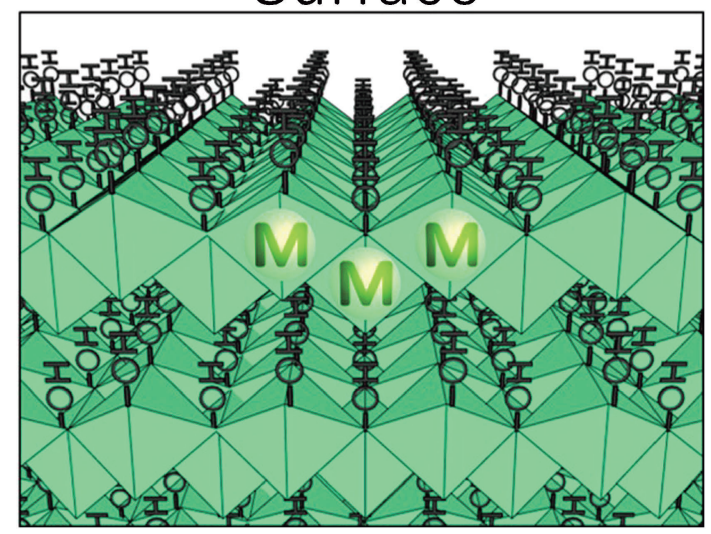

Fig. 1. Schematics showing conventional 1D (nanotube and nanobelt) and 2D (nanoplate) nanostructures of crystalline metal hydroxides (a) and the crystalline layered structure where hydroxyl groups are aligned in atomic level (b).

\section{Controlling orientation and aggregation of titanate nanotube}

\subsection{Titanate nanotube}

A titanate nanotube (TNT) is a tubular 1D nanomaterial with $5-10 \mathrm{~nm}$ in diameter and tens to several hundreds of nanometers in length. ${ }^{8)}$ The crystal structure of TNT is known to be a lepidocrocite-type structure composed of layered titanate containing cations in the interlayer, which is same as gamma-type metal (oxy)hydroxide such as $\gamma$ $\mathrm{AlOOH}, \gamma-\mathrm{FeOOH}$, and $\mathrm{Cu}(\mathrm{OH})_{2}$. Owing to the characters such as the layered structure capable of cation-exchange, physicochemical properties similar to titania, high aspect ratio, and the large specific surface area originated from the tubular morphology, TNT is considered as a promising material for (photo)catalysts, hydrogen and Li ion storages, electrodes for Li ion batteries and solar cells, and light and electron emitters. ${ }^{8)}$ TNTs are usually obtained as powders via a hydrothermal treatment (e.g., at $150^{\circ} \mathrm{C}$ for $24 \mathrm{~h}$ ) of crystalline $\mathrm{TiO}_{2}$ powders with $10 \mathrm{M} \mathrm{NaOH}$ aqueous solution where homogeneous $\mathrm{TiO}_{2}$ dissolution and TNT nucleation processes are generally out of control in a reaction solution, resulting in hairball-like secondary particles constituted of entangled TNTs with a lack of macroscopic orientation and aggregation. ${ }^{9)}$ Due to the TNT formation process based on homogeneous nucleation, controlling macroscopic orientation and aggregation of TNTs is rather difficult in the reported approaches.

\subsection{Controlling orientation of titanate nano- tubes for interfacial applications}

The author and collaborators reported an alternative approach based on inhomogeneous nucleation of TNTs on

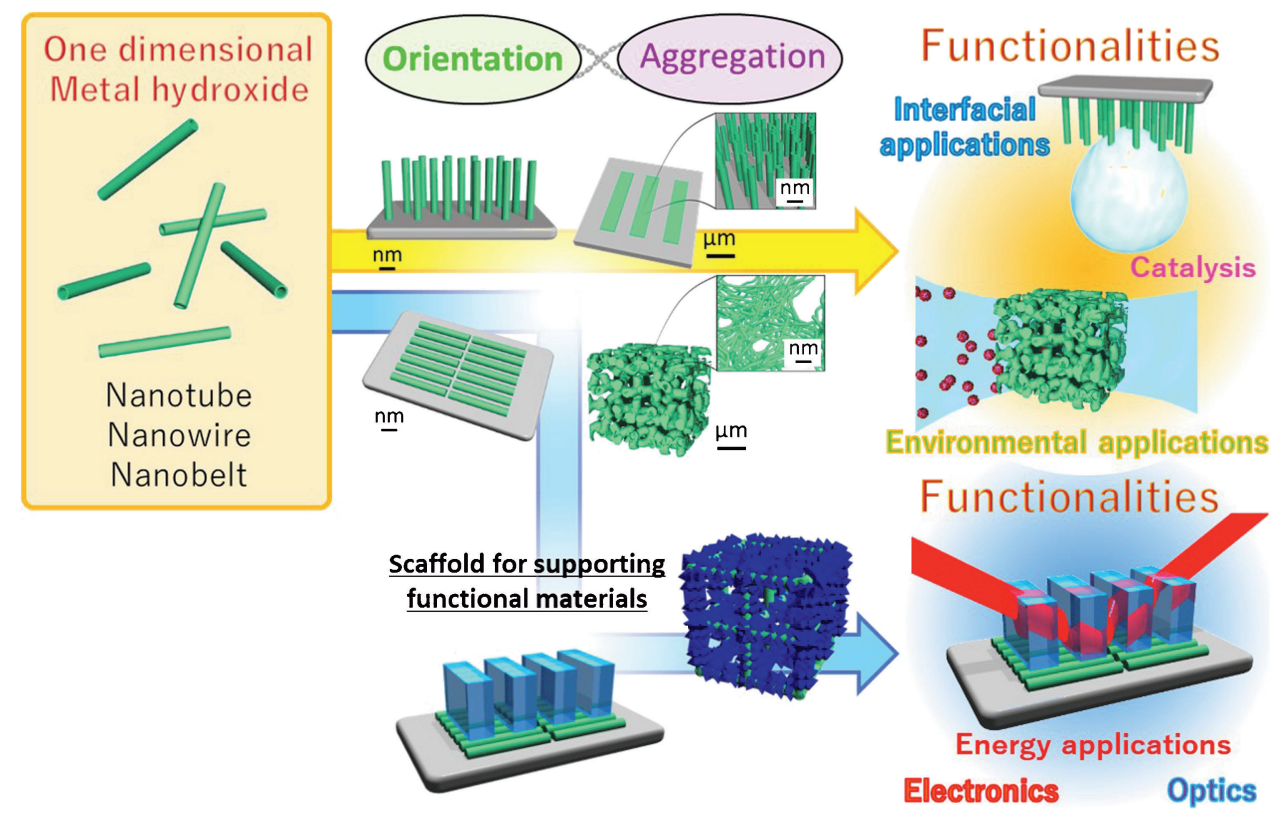

Fig. 2. Schematic showing two approaches for functionalization by controlling orientation and aggregation of 1D metal hydroxide nanomaterials. The approach indicated by a yellow arrow shows a direct functionalization by controlled orientation and aggregation of 1D metal hydroxide nanomaterials. The approach indicated by a blue arrow shows functionalization induced by the structuralization of other functional materials, MOF, derived from orientation/aggregation-controlled 1D metal hydroxide nanomaterials. 




(c)

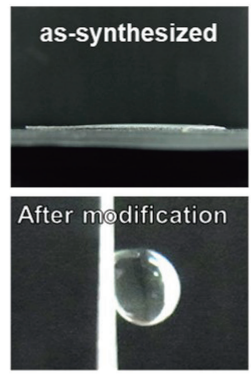

(b)

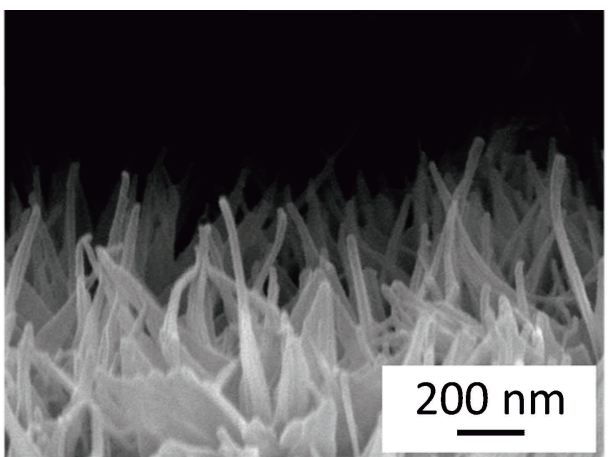

(d)



Fig. 3. Vertically-oriented titanate nanotubes on a precursory $\mathrm{TiO}_{2}$ film: an illustration (a) and a cross-sectional SEM image (b). (c) An $8 \mu \mathrm{l}$ water droplet on the vertically-oriented TNTs, as-prepared $\left(\mathrm{CA} \sim 0^{\circ}\right)$, after FAS modification $\left(\mathrm{CA}=152^{\circ}\right)$. Reproduced from Ref. 10 with permission from The Royal Society of Chemistry. (d) Reversible water adhesion of the FAS-modified vertically-oriented TNTs by alternating thermal-treatment $\left(80^{\circ} \mathrm{C}, 30 \mathrm{~min}\right)$ and hydration $(30 \mathrm{~min}$ in water). Optical images of the film catching and repelling a water droplet. Reproduced from Ref. 11 with permission from The Royal Society of Chemistry.

a precursory film to grow vertically-oriented TNTs on a substrate. ${ }^{10)}$ A sol-gel derived amorphous $\mathrm{TiO}_{2}$ film was used as a precursor for the TNT growth instead of the conventional powdery $\mathrm{TiO}_{2}$, and a relatively milder hydrothermal condition, for example $110^{\circ} \mathrm{C}$ in $<1 \mathrm{M} \mathrm{NaOH}$ aqueous solution, was employed because the amorphous $\mathrm{TiO}_{2}$ film exhibits higher solubility in a basic solution under hydrothermal conditions due to the low crystallinity and the existence of micro pores. ${ }^{10)}$ The employment of the sol-gel derived amorphous $\mathrm{TiO}_{2}$ film as a precursor and the relatively milder hydrothermal condition leaded to inhomogeneous nucleation of TNTs on the precursory $\mathrm{TiO}_{2}$ film, resulting in oriented growth of TNTs perpendicularly to the entire surface of the precursory $\mathrm{TiO}_{2}$ film [Figs. 3(a) and 3(b)]. As seen in the Fig. 3(b), the resultant TNTs has an inverse funnel shape, in which the tops of the nanosheets anchored on the precursory film surface are rolled-up to form nanotubes. In addition to the inhomogeneous nucleation approach affording the formation of the vertically-oriented TNTs, the use of the sol-gel derived amorphous $\mathrm{TiO}_{2}$ film and the relatively milder hydrothermal condition can allow for the fabrication of vertically-oriented TNTs on a variety of substrates including silica glasses, plastics, and rubbers. ${ }^{11)}$ Such semiconducting one-dimensional nanomaterials vertically-aligned on substrates are considered as promising electrodes for solar cells owing to a direct pathway for electron transfer from the point of injection to the electron sink with an improved electron transport and a higher charge collection efficiency. ${ }^{8), 12)}$

The macroscopically oriented 1D nanostructures and the abundant surface hydroxyl groups on TNTs enable to fabricate functional interfaces. ${ }^{10), 13)}$ The as-synthesized vertically-oriented TNTs exhibited superhydrophilic surface with a contact angle (CA) of $\sim 0^{\circ}$ [Fig. 3(c)]. When surface hydrophobizing agents such as fluoroalkylsilane (FAS) and long-chain carboxylic acid, which reduce surface free energy, were grafted onto the TNTs by a chemical vapor deposition method, the surface showed superhydrophobicity $\left(\mathrm{CA}=152^{\circ}\right)$. The superhydrophobic surface also exhibited strong water adhesion: an $8 \mu \mathrm{L}$ water droplet on the surface is strong enough to keep it anchored even while tilting the substrate 90 or $180^{\circ}$ (superhydrophobic adhesive surface). The strong water adhesion was found to be originated from physically adsorbed water on remaining hydroxyl groups of TNTs. A considerable amount of unmodified surfaces remained due to higher density of surface hydroxyl groups than the grafting density of the hydrophobizing agents on TNTs. The physically adsorbed water plays a crucial role as a glue for the water droplet. This finding allowed for reversible and switchable water adhesion on the superhydrophobic adhesive surface by controlling adsorption/desorption of the physically adsorbed water on the TNTs. Desorption of physically adsorbed water on TNTs takes place by a gentle heat treatment beyond $80^{\circ} \mathrm{C}$, and re-adsorption 
(hydration) recovers in an ambient atmosphere without any structural change of TNTs. The contact angle reversibly changed from $\sim 150$ to $\sim 137^{\circ}$ by alternating mild heating $\left(80^{\circ} \mathrm{C}, 30 \mathrm{~min}\right)$ and hydration $(30 \mathrm{~min}$ in water) [Fig. 3(d)]. ${ }^{11)}$ The change of contact angle enabled the surface repeatedly to catch and repel a falling water droplet $(8 \mu \mathrm{L})$. Such a superhydrophobic adhesive surface is potentially used for small water droplet manipulation without any loss in a lab-on-a-chip, microfluid devices and water/oil separation. ${ }^{14), 15)}$

\subsection{Controlling aggregation of titanate nano- tubes for monolithic cation-exchanger and photocatalyst}

TNT is one of potential candidate materials for solid catalysts, cation exchangers, and adsorbents for environmental toxic ions due to their high surface area, ${ }^{8)}$ strong Brønsted acidity, ${ }^{16}$ ) cation exchange capability ${ }^{17)}$ and photocatalytic activity. ${ }^{18)}$ Although TNTs are usually obtained in a powder form, bulk-shaped (monolithic) TNTs on the centimeter scale are usually preferred in practical applications such as catalysts, adsorbents, and hydrogen and $\mathrm{Li}^{+}$storage materials from a view point of handling, recovery, separation, and recycling. However, green pellets prepared by mechanical pressing of powdery TNTs are usually dense, resulting in an insufficient number of active sites to the surfaces of bulk materials because of the absence of flow channels inside the bulk materials for the rapid diffusion of adsorbate. ${ }^{19)}$ Hierarchically porous TNTs monoliths are preferable in these applications. The author and collaborators reported the fabrication of hierarchically porous TNT-based monoliths by interconnecting individual TNTs with titania nanoparticles (Fig. 4). ${ }^{20}$ A condensed aqueous emulsion of $\mathrm{TiO}_{2}$ nanoparticles was employed as precursors, which yielded TNT secondary particles and intervening TNT bridges within small cavities between the TNT secondary particles during the conversion from $\mathrm{TiO}_{2}$ to TNT. ${ }^{21)}$ The TNT bridges promote the formation of three-dimensional TNT frameworks [Figs. 4(c) and 4(d)]. The dense and entangled TNTs formed in small cavities resulted in other more sparse TNT areas. The TNT density variation generated continuous macropores as seen in Figs. 4(b) and 4(c). The resulting monolith has both nanopores derived from the nanotubular structure and macropores with a broad distribution from $10 \mathrm{~nm}$ to $10 \mu \mathrm{m}$ in diameter. It was found that the macro porosity is adjustable by simply varying the concentration of titania emulsions: smaller concentration of the emulsion resulted in larger porosity. The monolith has potential as a $\mathrm{Li}^{+}$storage material, an adsorbent for environmental toxic ions, and a reusable photocatalytic monolith owing to the hierarchical architecture, and cation-exchangeable and photocatalytic activities of TNT. $\mathrm{A} \mathrm{Li}^{+}$adsorption investigation showed that the monolith exhibited a faster rate and a higher capacity of $\mathrm{Li}^{+}$exchange than a dense TNT pellet [Fig. 4(e)]. The monolith is also useable as a recyclable photocatalytic monolith [Fig. 4(f)]. The photocatalytic activity was found to be enhanced by partially converting TNTs into $\mathrm{SrTiO}_{3}$ nanoparticles where $\mathrm{SrTiO}_{3}$ nanoparticles have a close contact with TNTs. ${ }^{22)}$

\section{Controlling orientation and aggregation of $\mathrm{Cu}(\mathrm{OH})_{2}$ nanotubes/nanobelts}

\section{1 $\mathrm{Cu}(\mathrm{OH})_{2}$ nanotube/nanobelt as a scaffold for fabricating MOF architectures}

Metal hydroxide has, in principle, lower lattice enthalpy than metal oxide, ${ }^{23)}$ and also abundant hydroxyl groups on its surface, which makes it a promising precursor and scaffold for organic-inorganic hybrid materials. As one of fascinating organic-inorganic hybrid materials, MOFs also called porous coordination polymers constituted of metal ion connectors and organic linkers receive a considerable attention as a new class of molecular-scale microporous materials due to their tunable architectures and chemical functionalities. $^{24), 25)}$ Due to these features, MOF attracts great interests for applications such as storage, separation and catalysis. ${ }^{26), 27)}$ In contrast, research interests for applications are now rapidly growing on MOF-based electrical/ optical devices including sensors, microelectronics, and transistors because their functionalities are designable in a molecular level. ${ }^{28)}$ Positioning MOF crystals at desired locations and controlling an orientation have been highly desired to realize MOF-based devices. ${ }^{29)}$ MOFs are usually synthesized by a solvothermal method from metal salt and organic linker. The author and collaborators have been developing a MOF fabrication method using metal hydroxide as a precursor and scaffold. ${ }^{30), 31)} \mathrm{Cu}(\mathrm{OH})_{2}$ nanotubes/nanobelts are mostly employed as scaffolds and $\mathrm{Cu}^{2+}$ sources for the MOF synthesis because of the existence of a variety of fascinating $\mathrm{Cu}$-based MOFs. The conversion from $\mathrm{Cu}(\mathrm{OH})_{2}$ into $\mathrm{Cu}$-based $\mathrm{MOF}$ is conducted by simply soaking the $\mathrm{Cu}(\mathrm{OH})_{2}$ nanotubes/nanobelts into a solution containing organic linkers constituting the target $\mathrm{Cu}$-based MOFs [Fig. 5(a)]. In this process, a direct reaction of $\mathrm{Cu}(\mathrm{OH})_{2}$ with the organic linkers takes place through an acid-base reaction. Owing to the high reactivity of $\mathrm{Cu}(\mathrm{OH})_{2}$ with the organic linkers originated from the low lattice enthalpy and the abundant reactive hydroxyl groups, this approach offers advantages such as short processing time and mild synthetic conditions (e.g., room temperature, mixed solvent of water and ethanol). The use of pre-deposited $\mathrm{Cu}(\mathrm{OH})_{2}$ nanotubes/nanobelts can also afford the fabrication of $\mathrm{Cu}$-based MOFs on 3D architectures. Figures 5(b)-5(d) show $\mathrm{Cu}_{3}(\mathrm{BTC})_{2}(\mathrm{BTC}=1,3,5-$ benzenetricarboxylate) MOF patterns fabricated from predeposited $\mathrm{Cu}(\mathrm{OH})_{2}$ nanotube arrays. ${ }^{32}$ The $\mathrm{Cu}(\mathrm{OH})_{2}$ nanotube arrays were synthesized by surface oxidation of metallic copper patterns in a basic solution. The protocol also allows to homogeneously decorate a $\mathrm{Cu}$ mesh with the $\mathrm{Cu}_{3}(\mathrm{BTC})_{2}$ MOFs [Figs. 5(e) and 5(f)]. The MOFdecorated mesh is usable as a supported catalyst to accelerate the Friedländer reaction between 2-aminobenzophenones and acetylacetone [Fig. 5(g)]. The MOF films on a various substrates are also fabricated from $\mathrm{Cu}(\mathrm{OH})_{2}$ nanobelt films prepared by depositing a $\mathrm{Cu}(\mathrm{OH})_{2}$ nanobelt dispersion. The MOF films on conductive substrates can 

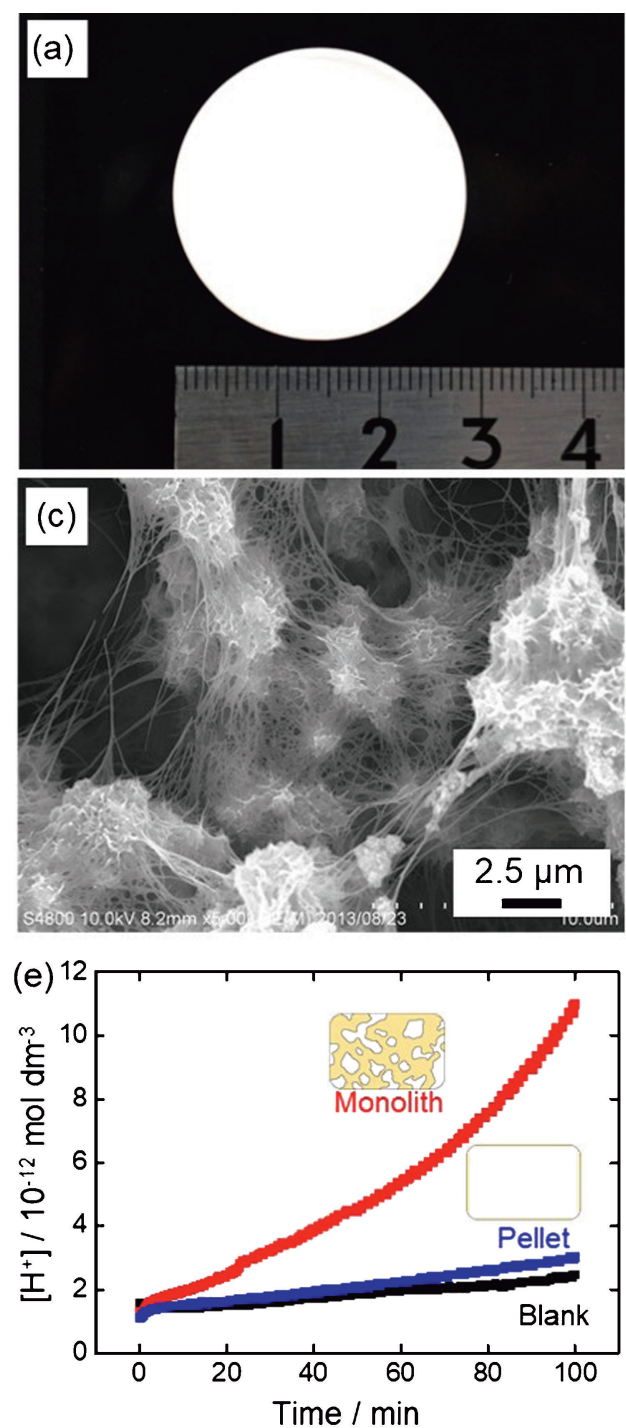

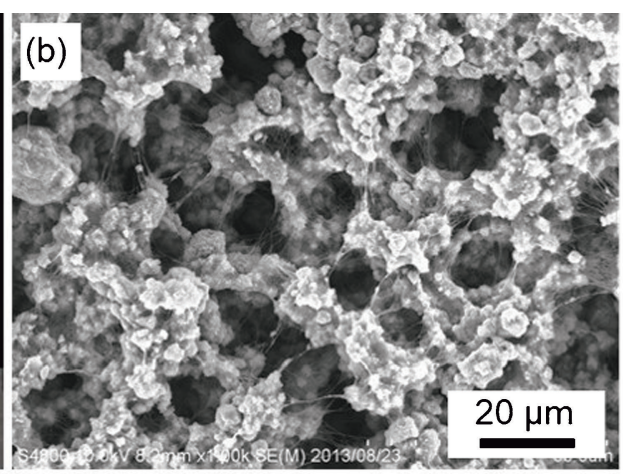

(d)
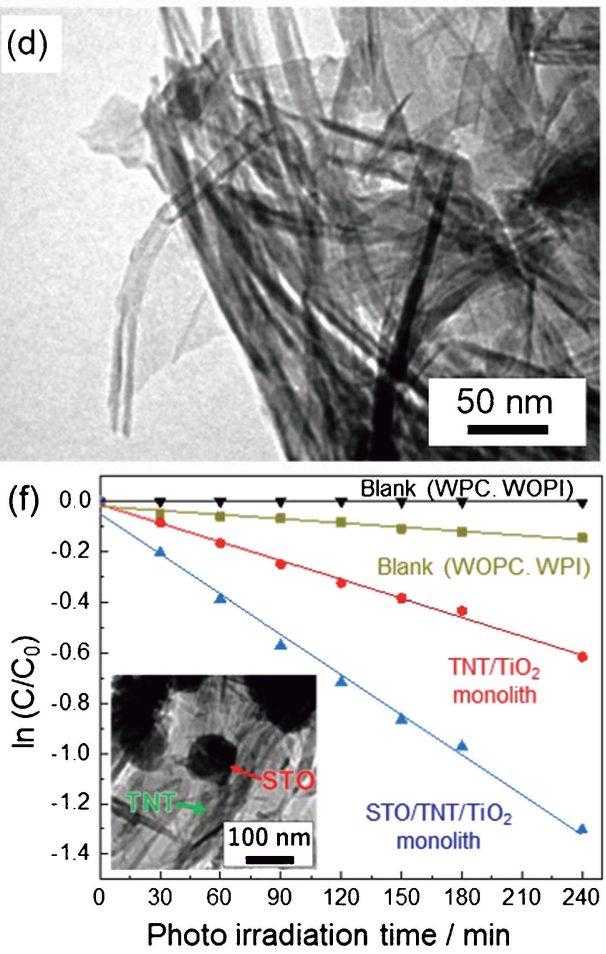

Fig. 4. Hierarchically porous TNT-based monolith: (a) Optical image of the centimeter-scale monolith. (b, c) SEM images showing the macropores and framework constructed of interconnected and entangled TNTs. TNT secondary particles were bridged by entangled TNTs. (d) Transmission electron microscope (TEM) image showing the tubular TNT structure. (e) Lithium-proton ion exchange capability of hierarchically porous TNTbased monolith, dense pellet and blank. Reproduced from Ref. 20 with permission from American Chemical Society. (f) Photocatalytic degradation (First-order reaction kinetics for the rhodamine B (RhB) degradation) by the TNT-based (TNT/TiO $)$ monolith and the TNT-based monolith partially converted to $\mathrm{SrTiO}_{3}(\mathrm{STO} / \mathrm{TNT} /$ $\mathrm{TiO}_{2}$ monolith). Blank (WPC. WOPI) indicates bank data collected with photocatalyst without photoirradiation, and Blank (WOPC. WPI) indicates data collected without photocatalyst with photoirradiation. Reproduced from Ref. 22 with permission from The Ceramic Society of Japan.

be used for size-selective electrochemical sensing and nonenzymatic electrocatalysis of glucose. ${ }^{33)}$

\subsection{Oriented $\mathrm{Cu}(\mathrm{OH})_{2}$ nanobelts as scaffolds for fabricating MOF oriented films}

An achievement of MOF films with macroscopically controlled crystallographic orientations has been required to enhance the sophisticated functionalities for practical applications. As one of promising methods, liquid phase epitaxy on surfaces functionalized with self-assembled monolayer has been used for oriented MOF films. ${ }^{34)}$ However, a control of crystallographic orientation along all three axes in a macroscopic scale was not achieved; especially, in-plane orientation (a direction parallel to a substrate) is hardly controlled by reported methods for oriented MOF film. The author and collaborators have recently developed a novel method achieving MOF films with crystallographic orientation along all three axes in a centimetre-scale via heteroepitaxial growth of MOF on metal hydroxide. ${ }^{35)}$ The key to success is the use of metal hydroxide as a scaffold and precursor [Figs. 6(a) and 6(b)]. As described above, metal hydroxide has aligned hydroxyl groups on the surface in atomic level, which differ from metal oxide and other materials. The surface 
(a)


(e)
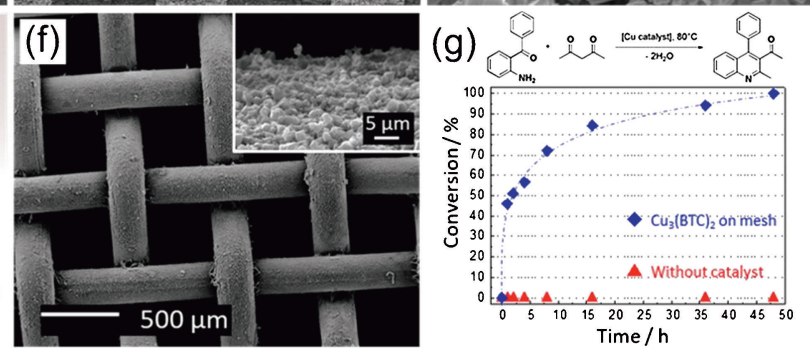

Fig. 5. (a) Illustration representing a conversion from metal hydroxide to MOF. (b-c) SEM images of $\mathrm{Cu}_{3}(\mathrm{BTC})_{2}$ MOF patterns in which the white parts are decorated with MOF crystals. (e) Optical image of a $\mathrm{Cu}$ mesh (left), a $\mathrm{Cu}$ mesh decorated with $\mathrm{Cu}(\mathrm{OH})_{2}$ nanotubes (middle), and a $\mathrm{Cu}$ mesh decorated with MOFs (right). (f) SEM image of the $\mathrm{Cu}$ mesh decorated with MOFs. (g) Friedländer reaction conversion (\%) performed for $\mathrm{Cu}_{3}(\mathrm{BTC})_{2}$ on copper mesh and without any catalyst. Reproduced from Ref. 32 with permission from Wiley$\mathrm{VCH}$.

hydroxyl groups are able to be directly reacted with carboxylic acid-based organic linkers through an acid-base reaction, possibly resulting in the aligned organic linker layers on the surface of metal hydroxide. Given that MOFs are constructed via a modular synthetic approach we hypothesized that an epitaxially aligned MOF would be synthesized if the dimensions of the organic linkers were analogous to the lattice parameter (or a whole number multiple) of the metal hydroxide: heteroepitaxial growth takes place in case the regularity of the organic linkers of MOF is consisted with that of hydroxyl groups of metal hydroxide [Fig. 6(c)]. By consulting a database, it was found that $\mathrm{Cu}_{2}(\mathrm{BDC})_{2}$ (BDC: 1,4-benzenedicarboxylate) possesses lattice parameters ( $a$ and $b$ axes) that closely match those of $\mathrm{Cu}(\mathrm{OH})_{2}$ ( $c$ and $a$ axes): $P 4$ space group, $a=10.61 \AA, b=5.80 \AA, c=10.61 \AA$ in $\mathrm{Cu}_{2}(\mathrm{BDC})_{2}$, and Cmc21 space group, $a=2.95 \AA, b=10.59 \AA, c=5.26 \AA$ in $\mathrm{Cu}(\mathrm{OH})_{2}$. Thus, we predicted that the $a$ and $b$ axis of the MOF would align with the $c$ and $a$ axis of a $\mathrm{Cu}(\mathrm{OH})_{2}$ substrate, respectively. An oriented $\mathrm{Cu}(\mathrm{OH})_{2}$ nanobelt film is employed as a "pseudo single crystal film" because the synthesis of large single crystal $\mathrm{Cu}(\mathrm{OH})_{2}$ is almost impossible due to the rapid crystallization [Fig. 6(d)]. Figures 6(e) and 6(f) exhibit the $\mathrm{Cu}_{2}(\mathrm{BDC})_{2}$ MOFs grown on the pseudo single crystal film of $\mathrm{Cu}(\mathrm{OH})_{2}$. The scanning electron microscope (SEM) images clearly show that the plate $\mathrm{Cu}_{2}(\mathrm{BDC})_{2}$ crystals aligned in a same direction orthogonal to the nanobelts. The crystallographic orientation along all three axes in a centimetre-scale was confirmed by X-ray diffraction investigations. The macroscopically oriented MOF films are capable of introducing functional molecules into their micropores for generating anisotropic physical properties. The MOF films accommodating organic dye molecules exhibited the switchable fluorescence 'ON' or 'OFF' by changing the orientation of the substrate with respect to the polarization direction of the incident light [Figs. 6(g)-6(i)]. The result confirmed that the oriented MOF film can afford a macroscopic alignment of functional molecules in a same direction. By applying liquid phase epitaxy on the oriented MOF films, the precise alignment of multiple layers of MOF films, MOF-on-MOF films, over macroscopic length scales can be fabricated, providing a new toolbox for multifunctional microporous coatings. ${ }^{36)}$

\section{Summary}

Recent advances on the fabrication methods affording macroscopically controlled orientation and aggregation of 1D metal hydroxide nanomaterials and the structuralizationdriven applications were overviewed with an emphasis on the author's works. The fabrication of vertically-oriented TNTs leaded to superhydrophobic adhesive surfaces. By 
(a)

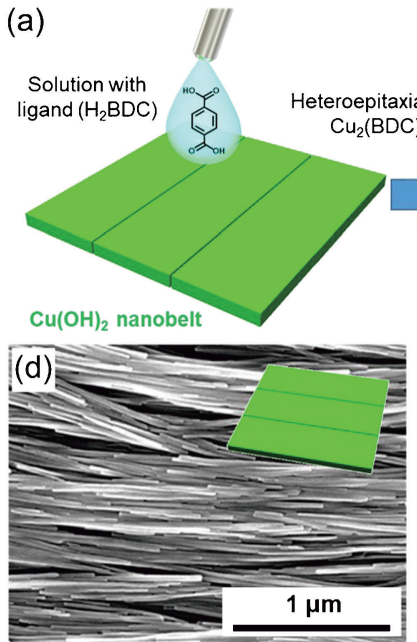

(g)



(b)
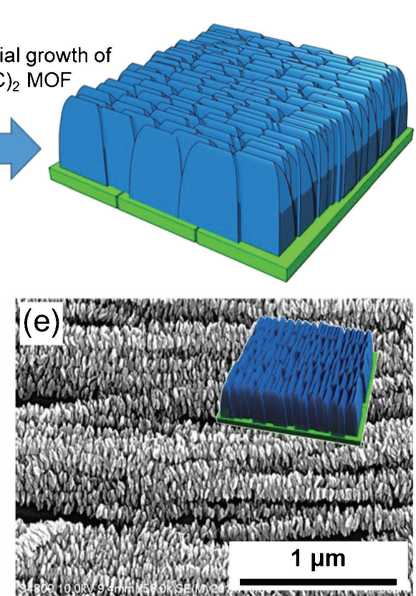

(h)

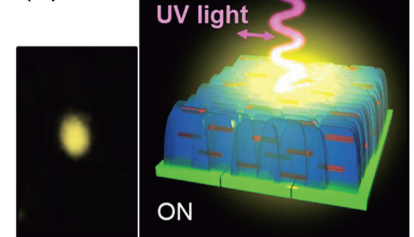

(c)
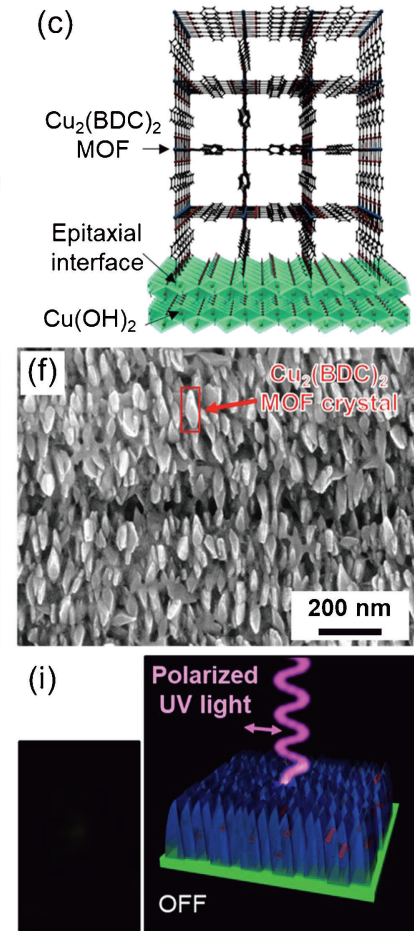

Fig. 6. (a-c) Schematics representing heteroepitaxial growth of $\mathrm{Cu}_{2}(\mathrm{BDC})_{2} \mathrm{MOF}$ on $\mathrm{Cu}(\mathrm{OH})_{2}$. (d) SEM image of a oriented $\mathrm{Cu}(\mathrm{OH})_{2}$ nanobelt film. (e, f) SEM images of $\mathrm{Cu}_{2}(\mathrm{BDC})_{2}$ MOF film grown on the oriented $\mathrm{Cu}(\mathrm{OH})_{2}$ nanobelt film. (g) illustration of oriented MOF film accommodating dye \{4-[4-(dimethylamino)-styryl]pyridine, DMASP . Fluorescent spot images where polarization of the excitation light is parallel (ON state) (h) and perpendicular (OFF state) (i) to a transition dipole moment (polarization axis) of DMASP. Reproduced from Ref. 35 with permission from Nature Publishing Group.

utilizing water adsorption/desorption characters on the TNTs, the switchable wettability on the superhydrophobic adhesive surfaces was achieved. Hierarchically porous TNT-based monoliths were fabricated by interconnecting individual TNTs, which are demonstrated to be usable as reusable cation-exchanging and photocatalytic monoliths. Metal hydroxide can be also a promising scaffold and precursor for positioning MOF crystals at desired locations and controlling an orientation owing to low lattice enthalpy and aligned and abundant reactive hydroxyl groups originated from the nature of metal hydroxide. To our best knowledge, heteroepitaxial growth of MOF on metal hydroxide is a first report fruitfully utilizing the aligned hydroxyl groups on metal hydroxide in atomic level. The fruitful use of the aligned hydroxyl groups is expected to be extended to the other organic-inorganic hybrid materials, organics, and inorganics.

Acknowledgements The author is grateful to Prof. Masahide Takahashi (Osaka Prefecture University) for his continuous support, intense discussion, and encouragement. The author would like to thank to Profs. Paolo Falcaro (Graz University of Technology), Christian Doonan (The University of Adelaide), Yasuaki Tokudome and Atsushi Nakahira (Osaka Prefecture University) for their valuable advices and sincere supports to conceive the present study. Special thanks go to coworkers, collaborators, and students for their contributions to this work. Financial supports from JSPS KAKENHI and JST PRESTO Programs (JPMJPR19I3) are gratefully acknowledged.

\section{Reference}

1) E. Roduner, Chem. Soc. Rev., 35, 583-592 (2006).

2) C. Burda, X. Chen, R. Narayanan and M. A. El-Sayed, Chem. Rev., 105, 1025-1102 (2005).

3) H. Yin and Z. Tang, Chem. Soc. Rev., 45, 4873-4891 (2016).

4) M. Takemoto, Y. Tokudome, S. Kikkawa, K. Teramura, T. Tanaka, K. Okada, H. Murata, A. Nakahira and M. Takahashi, RSC Adv., 10, 8066-8073 (2020).

5) J. H. Choy, S. Y. Kwak, J. S. Park, Y. J. Jeong and J. Portier, J. Am. Chem. Soc., 121, 1399-1400 (1999).

6) N. Tarutani, Y. Tokudome, M. Fukui, K. Nakanishi and M. Takahashi, RSC Adv., 5, 57187-57192 (2015).

7) Q. Wang and D. Ohare, Chem. Rev., 112, 4124-4155 (2012).

8) D. V. Bavykin and F. C. Walsh, Eur. J. Inorg. Chem., 2009, 977-997 (2009).

9) T. Kasuga, M. Hiramatsu, A. Hoson, T. Sekino and K. Niihara, Adv. Mater., 11, 1307-1311 (1999).

10) K. Okada, Y. Tokudome, P. Falcaro, Y. Takamatsu, A. Nakahira and M. Takahashi, Chem. Commun., 48, 6130-6132 (2012).

11) Y. Tokudome, K. Okada, A. Nakahira and $M$. Takahashi, J. Mater. Chem. A, 2, 58-61 (2014).

12) D. V. Bavykin, J. M. Friedrich and F. C. Walsh, $A d v$. Mater., 18, 2807-2824 (2006).

13) K. Okada, Y. Tokudome and M. Takahashi, J. Sol-Gel 
Sci. Techn., 79, 389-394 (2016).

14) K. Tsougeni, D. Papageorgiou, A. Tserepi and E. Gogolides, Lab Chip, 10, 462-469 (2010).

15) D. Wang, Y. Liu, X. Liu, F. Zhou, W. Liu and Q. Xue, Chem. Commun., 7018-7020 (2009).

16) M. Kitano, E. Wada, K. Nakajima, S. Hayashi, S. Miyazaki, H. Kobayashi and M. Hara, Chem. Mater., 25, 385-393 (2013).

17) R. Yoshida, Y. Suzuki and S. Yoshikawa, Mater. Chem. Phys., 91, 409-416 (2005).

18) J. S. Jang, S. H. Choi, D. H. Kim, J. W. Jang, K. S. Lee and J. S. Lee, J. Phys. Chem. C, 113, 8990-8996 (2009).

19) A. Nakahira, T. Kubo and Y. Yamasaki, ACS Appl. Mater. Interfaces, 2, 1136-1140 (2010).

20) K. Okada, G. Asakura, Y. Tokudome, A. Nakahira and M. Takahashi, Chem. Mater., 27, 1885-1891 (2015).

21) K. Okada, Y. Takamatsu, Y. Tokudome, A. Nakahira and M. Takahashi, J. Sol-Gel Sci. Techn., 65, 36-40 (2013).

22) K. Okada and M. Takahashi, J. Ceram. Soc. Jpn., 127, 761-766 (2019).

23) W. M. Haynes, "CRC Handbook of Chemistry and Physics: A Ready-Reference Book of Chemical and Physical Data", CRC Press (2011).

24) S. Kitagawa, R. Kitaura and S. Noro, Angew. Chem., Int. Ed. Engl., 43, 2334-2375 (2004).

25) O. M. Yaghi, M. O'Keeffe, N. W. Ockwig, H. K. Chae, M. Eddaoudi and J. Kim, Nature, 423, 705-714 (2003).

26) M. Eddaoudi, J. Kim, N. Rosi, D. Vodak, J. Wachter, M. O'Keeffe and O. M. Yaghi, Science, 295, 469-472
(2002)

27) J. Lee, O. K. Farha, J. Roberts, K. A. Scheidt, S. T. Nguyen and J. T. Hupp, Chem. Soc. Rev., 38, 14501459 (2009).

$28)$ I. Stassen, N. Burtch, A. Talin, P. Falcaro, M. Allendorf and R. Ameloot, Chem. Soc. Rev., 46, 3185-3241 (2017).

29) P. Falcaro, R. Ricco, C. M. Doherty, K. Liang, A. J. Hill and M. J. Styles, Chem. Soc. Rev., 43, 5513-5560 (2014)

30) K. Ikigaki, K. Okada, Y. Tokudome and M. Takahashi, J. Sol-Gel Sci. Techn., 89, 128-134 (2019).

31) T. Toyao, K. Liang, K. Okada, R. Ricco, M. J. Styles, Y. Tokudome, Y. Horiuchi, A. J. Hill, M. Takahashi, M. Matsuoka and P. Falcaro, Inorg. Chem. Front., 2, 434441 (2015).

32) K. Okada, R. Ricco, Y. Tokudome, M. J. Styles, A. J. Hill, M. Takahashi and P. Falcaro, Adv. Funct. Mater, 24, 1969-1977 (2014).

33) K. Okada, S. Sawai, K. Ikigaki, Y. Tokudome, P. Falcaro and M. Takahashi, CrystEngComm, 19, 41944200 (2017).

34) J. L. Zhuang, A. Terfort and C. Wöll, Coordin. Chem. Rev., 307, 391-424 (2016).

35) P. Falcaro, K. Okada, T. Hara, K. Ikigaki, Y. Tokudome, A. W. Thornton, A. J. Hill, T. Williams, C. Doonan and M. Takahashi, Nat. Mater., 16, 342-348 (2017).

36) K. Ikigaki, K. Okada, Y. Tokudome, T. Toyao, P. Falcaro, C. J. Doonan and M. Takahashi, Angew. Chem. Int. Edit., 58, 6886-6890 (2019).

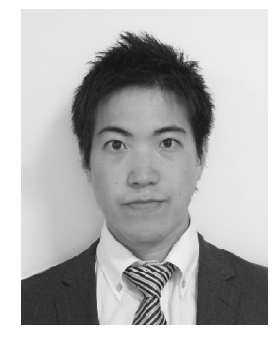

Kenji Okada received his Ph.D (Engineering) in Material Science from Osaka Prefecture University in 2014. After receiving the degree, he was funded as a JSPS (Japan Society for the Promotion of Science) postdoctoral researcher from 2014 to 2015. In 2015, he was appointed as an assistant professor at Osaka University. He moved to Osaka Prefecture University as an assistant professor in 2017. He was awarded as 'Leading Initiative for Excellent Young Researchers (LEADER)' by MEXT in 2017. He has been a PRESTO researcher of JST since 2019. His research interest is to fabricate nanomaterials with macroscopically controlled orientation and aggregation for electrical, optical and thermal applications. 